\title{
Zero-Hopf bifurcation in a Chua system
}

\author{
Rodrigo D. Euzébio ${ }^{\mathrm{a}, \mathrm{b}, *}$, Jaume Llibre ${ }^{\mathrm{b}}$ \\ a Departament de Matemática, IBILCE, UNESP, Rua Cristovao Colombo 2265, Jardim Nazareth, \\ CEP 15.054-00, Sao José de Rio Preto, SP, Brazil \\ ${ }^{\mathrm{b}}$ Departament de Matemàtiques, Universitat Autònoma de Barcelona, 08193 Bellaterra, Barcelona, \\ Catalonia, Spain
}

\section{A R T I C L E I N F O}

\section{Article history:}

Received 26 November 2014

Accepted 4 February 2017

Available online 6 March 2017

\section{Keywords:}

Chua system

Periodic orbit

Averaging theory

Zero Hopf bifurcation

\begin{abstract}
A B S T R A C T
A zero-Hopf equilibrium is an isolated equilibrium point whose eigenvalues are $\pm \omega i \neq 0$ and 0 . In general for a such equilibrium there is no theory for knowing when it bifurcates some small-amplitude limit cycles moving the parameters of the system. Here we study the zero-Hopf bifurcation using the averaging theory. We apply this theory to a Chua system depending on 6 parameters, but the way followed for studying the zero-Hopf bifurcation can be applied to any other differential system in dimension 3 or higher.

In this paper first we show that there are three 4-parameter families of Chua systems exhibiting a zero-Hopf equilibrium. After, by using the averaging theory, we provide sufficient conditions for the bifurcation of limit cycles from these families of zero-Hopf equilibria. From one family we can prove that 1 limit cycle bifurcates, and from the other two families we can prove that 1, 2 or 3 limit cycles bifurcate simultaneously.
\end{abstract}

() 2017 Elsevier Ltd. All rights reserved.

\section{Introduction and statement of the main results}

The Chua system is a classical model for electronic circuit and one of the most simplest models presenting chaos. It was presented by Chua, Komuro and Matsumoto [1] in 1986 and exhibits a rich range of dynamical behavior. There are several different models of Chua's systems see for instance [2-6].

The Chua circuit considered in [1] is a relaxation oscillator with a cubic nonlinear characteristic. It can be thought as a circuit comprising a harmonic oscillator for which the operation is based on a field-effect transistor, coupled to a relaxation oscillator composed of a tunnel diode. The Chua system can be described

\footnotetext{
* Corresponding author at: Departament de Matemática, IBILCE, UNESP, RuaCristovao Colombo 2265, Jardim Nazareth, CEP 15.054-00, Sao José de Rio Preto, SP, Brazil.

E-mail addresses: euzebio@ime.unicamp.br, rodrigo.euzebio@sjrp.unesp.br (R.D. Euzébio), jllibre@mat.uab.cat(J. Llibre).
} 
by the following equations

$$
\begin{aligned}
& \frac{d x}{d t}=a\left(z-b x-a_{2} x^{2}-a_{1} x^{3}\right), \\
& \frac{d y}{d t}=-z, \\
& \frac{d z}{d t}=-b_{1} x+y+b_{2} z .
\end{aligned}
$$

Note that it depends on six parameters $a, a_{1}, a_{2}, b, b_{1}$ and $b_{2}$.

In [7] the authors analyze the existence of local and global analytic first integrals in the Chua system. In [8] the authors use techniques of Differential Geometry in order to obtain an analytical expression of the slow manifold equation of Chua system. In [9] was studied the dynamics at infinity of the Chua system for the particular case where $b_{1}$ and $b_{2}$ are both one. Besides, we can find some aspects about the Hopf bifurcation in $[10,11]$. In this paper, by using averaging theory, we study the limit cycles that can bifurcate from zero-Hopf equilibrium points of the Chua system (1). We note that at these points for our system (1) we can apply neither the classical Hopf bifurcation theory which needs that the real eigenvalue be non-zero, nor the standard theory developed up to now for some special cases of zero-Hopf equilibrium points as the ones analyzed in the papers [12-15]. A possible approach for studying the zero-Hopf equilibrium is to pass to normal form, but this needs some work. Here we shall show how to study zero-Hopf bifurcations directly without needing to pass to normal form. Other authors also have studied the zero-Hopf bifurcation in other Chua systems different from the Chua system here analyzed, see for instance the articles $[11,10,16]$ where $\dot{y}=x-y-z$, among other differences with the system (1).

The Chua system (1) can have at most three equilibria, namely: the origin and the two equilibria

$$
p_{ \pm}=\left(\frac{-a_{2} \pm \sqrt{a_{2}^{2}-4 a_{1} b}}{2 a_{1}},-\frac{a_{2} b_{1}}{2 a_{1}} \pm \frac{b_{1} \sqrt{a_{2}^{2}-4 a_{1} b}}{2 a_{1}}, 0\right),
$$

if $a_{2}^{2}-4 a_{1} b>0$ and $a_{1} \neq 0$. When $a_{2}^{2}-4 a_{1} b=0$ and $a_{1} a_{2} \neq 0$ the system has only two equilibria, the origin and the equilibrium

$$
p=\left(\frac{-a_{2}}{2 a_{1}},-\frac{a_{2} b_{1}}{2 a_{1}}, 0\right) .
$$

Otherwise the origin is the unique equilibrium of the system.

As far as we know, the study of existence or non-existence of zero-Hopf equilibria and zero-Hopf bifurcation in the Chua system has not been considered in the literature. In this paper we have this objective. The method used here for studying the zero-Hopf bifurcation can be applied to any differential system in $\mathbb{R}^{3}$.

A zero-Hopf equilibrium is an equilibrium point of a 3-dimensional autonomous differential system which has a zero eigenvalue and a pair of purely imaginary eigenvalues. In general the zero-Hopf bifurcation is a 2-parameter unfolding of a 3-dimensional autonomous differential system with a zero-Hopf equilibrium. The unfolding has an isolated equilibrium with a zero eigenvalue and a pair of purely imaginary eigenvalues if the two parameters take zero values and the unfolding has different dynamics in a small neighborhood of this isolated equilibrium as the two parameters vary in a small neighborhood of the origin. To read more about zero-Hopf bifurcation, see Guckenheimer, Han, Holmes, Kuznetsov, Marsden and Scheurle in [17,14,18-20]. Moreover, complex phenomena can occur at an isolated zero-Hopf equilibrium, as bifurcation of complicated invariant sets of the unfolding and a local birth of "chaos", as can be seen in the work of Baldomá and Seara, Broer and Vegter, Champneys and Kirk, Scheurle and Marsden in [21-24,20].

In the next proposition we characterize the Hopf equilibria of the Chua system. 
Proposition 1. There are three 4-parameter families of Chua systems having a zero-Hopf equilibrium point, one for the equilibrium point located at the origin and the other two for each one of the equilibria $p_{ \pm}$when they exist. Namely,

(a) $b=b_{2}=0$ and $a b_{1}+1>0$ for the origin; and

(b) $b=a_{2}^{2} /\left(4 a_{1}\right), b_{2}=0, a b_{1}+1>0, a_{2}^{2}-4 a_{1} b>0$ and $a_{1} \neq 0$ for $p_{ \pm}$.

The next result gives sufficient conditions for the bifurcation of a limit cycle from the origin when it is a zero-Hopf equilibrium.

Theorem 2. Let

$$
\left(a, a_{1}, a_{2}, b, b_{1}, b_{2}\right)=\left(\bar{a}_{0}+\varepsilon \alpha_{0}, \bar{a}_{1}+\varepsilon \alpha_{1}, \bar{a}_{2}+\varepsilon \alpha_{2}, \varepsilon \beta_{0}, \frac{\omega^{2}-1}{a}+\varepsilon \beta_{1}, \varepsilon \beta_{2}\right) .
$$

If $\bar{a}_{0} \bar{a}_{2} \neq 0,|\omega| \neq 0,1$ and

$$
\Gamma=\left(\bar{a}_{0} \beta_{0}\left(1-\omega^{2}\right)+\beta_{2} \omega^{2}\right)\left(\bar{a}_{0} \beta_{0} \omega^{2}\left(1-\omega^{2}\right)+\beta_{2} \omega^{4}\right)>0,
$$

then for $\varepsilon>0$ sufficiently small the Chua system has a zero-Hopf bifurcation at the equilibrium point located at the origin of coordinates, and a limit cycle appears at this equilibrium when $\varepsilon=0$. Moreover, this limit cycle has the same kind of stability or instability than an equilibrium point of a planar differential system with eigenvalues

$$
\frac{-\beta_{2} \omega^{5} \pm \sqrt{\omega^{6}\left(\beta_{2}^{2} \omega^{4}\left(3-2 \omega^{2}\right)+2 \bar{a}_{0}^{2} \beta_{0}^{2}\left(\omega^{2}-1\right)^{3}\right)}}{2 \omega^{6}\left(\omega^{2}-1\right)} .
$$

The following result provides sufficient conditions for the bifurcation of a limit cycle from the equilibrium $p_{-}$when it is zero-Hopf equilibrium.

Theorem 3. Consider the vector $\left(a, a_{1}, a_{2}, b, b_{1}, b_{2}\right)$ given by

$$
\begin{aligned}
& a=\bar{a}_{0}+\varepsilon \alpha_{0}+\varepsilon^{2} \xi_{0}, \\
& a_{1}=\bar{a}_{1}+\varepsilon \alpha_{1}+\varepsilon^{2} \xi_{1}, \\
& a_{2}=\varepsilon \alpha_{2}+\varepsilon^{2} \xi_{2}, \\
& b=\frac{a_{2}^{2}}{4 a_{1}}+\varepsilon^{2} \zeta_{0}, \\
& b_{1}=\frac{\omega^{2}-1}{a}+\varepsilon \beta_{1}+\varepsilon^{2} \zeta_{1}, \\
& b_{2}=\varepsilon^{2} \zeta_{2} .
\end{aligned}
$$

If $\bar{a}_{1} \omega \neq 0$ and $\bar{a}_{1} \zeta_{0}<0$ then, for $\varepsilon>0$ sufficiently small the Chua system has a zero-Hopf bifurcation at the equilibrium point located at $p_{-}$and three limit cycles can bifurcate from this equilibrium when $\varepsilon=0$. Moreover, examples of systems where 1, 2 or 3limit cycles bifurcate simultaneously are given.

Proposition 1 and Theorems 2 and 3 are proved in Section 3. In particular, both theorems are proved using the averaging method. This method will be briefly summarized in the next section. We note that Theorem 2 is proved using averaging theory of first order, but the proof of Theorem 3 needs averaging of second order.

Also the stability or instability of the bifurcated limit cycles in Theorem 3 can be studied, but the expressions of the eigenvalues which provide such stability or instability are huge and we do not give them here. 
Remark 1. For the equilibrium point $p_{+}$we have analogous results to the ones of Theorem 3 for $p_{-}$. For this reason, we omit the statement of the result for the equilibrium $p_{+}$and its proof. This fact is not due to any symmetry of the Chua system, it is only due to the fact that doing the corresponding computations for the equilibrium point $p_{+}$we obtain the same results than in the equilibrium $p_{-}$.

\section{Limit cycles via averaging theory}

The averaging method is a classical tool in nonlinear analysis and dynamical systems. The procedure of averaging can be found already in the work of Lagrange [25] and Laplace [26] who provided an intuitive justification of the method. After them, Poincaré considered the determination of periodic solutions by series expansion with respect to a small parameter, but until around 1930 we see the start of precise statements and proofs in averaging theory. After this time many new results in the theory of averaging have been obtained. The main contribution in direction to the formalization of the method started with Appleton and van der Pol [27] and Fatou [28], and later with the work of Bogoliubov and Krylov [29] and Bogoliubov [30].

Now we present the basic results on the averaging theory of first and second order. The averaging of first order for studying periodic orbits can be found in [31], see Theorems 11.5 and 11.6. It can be summarized as follows.

Theorem 4. We consider the following two initial value problems

$$
\dot{x}=\varepsilon f(t, x)+\varepsilon^{2} g(t, x, \varepsilon), \quad x(0)=x_{0},
$$

and

$$
\dot{y}=\varepsilon f^{0}(y), \quad y(0)=x_{0}
$$

where $x, y, x_{0} \in \Omega$ an open subset of $\mathbb{R}^{n}, t \in[0, \infty), \varepsilon \in\left(0, \varepsilon_{0}\right], f$ and $g$ are periodic of period $T$ in the variable $t$, and $f^{0}(y)$ is the averaged function of $f(t, x)$ with respect to $t$, i.e.,

$$
f^{0}(y)=\frac{1}{T} \int_{0}^{T} f(t, y) d t
$$

Suppose:

(i) $f$, its Jacobian $\frac{\partial f}{\partial x}$, its Hessian $\frac{\partial^{2} f}{\partial x^{2}}, g$ and its Jacobian $\frac{\partial g}{\partial x}$ are defined, continuous and bounded by a constant independent on $\varepsilon$ in $[0, \infty) \times \Omega$ and $\varepsilon \in\left(0, \varepsilon_{0}\right]$;

(ii) $T$ is a constant independent of $\varepsilon$; and

(iii) $y(t)$ belongs to $\Omega$ on the interval of time $[0,1 / \varepsilon]$. Then the following statements hold.

(a) On the time scale $1 / \varepsilon$ we have that $x(t)-y(t)=O(\varepsilon)$, as $\varepsilon \rightarrow 0$.

(b) If $p$ is a singular point of the averaged system (5) such that the determinant of the Jacobian matrix

$$
\left.\frac{\partial f^{0}}{\partial y}\right|_{y=p}
$$

is not zero, then there exists a limit cycle $\phi(t, \varepsilon)$ of period $T$ for system (4) which is close to $p$ and such that $\phi(0, \varepsilon) \rightarrow p$ as $\varepsilon \rightarrow 0$.

(c) The stability or instability of the limit cycle $\phi(t, \varepsilon)$ is given by the stability or instability of the singular point $p$ of the averaged system (5). In fact, the singular point $p$ has the stability behavior of the Poincaré map associated to the limit cycle $\phi(t, \varepsilon)$.

The next result presents the second order averaging method of a periodic differential system. For a proof see Theorem 3.5.1 of Sanders and Verhulst in [31], see also [32]. 
Theorem 5. We consider the following two initial value problems

$$
\dot{x}=\varepsilon f(t, x)+\varepsilon^{2} g(t, x)+\varepsilon^{3} R(t, x, \varepsilon), \quad x(0)=x_{0}
$$

and

$$
\dot{y}=\varepsilon f^{0}(y)+\varepsilon^{2}\left(f^{10}(y)+g^{0}(y)\right), \quad y(0)=x_{0},
$$

with $f, g:[0, \infty) \times \Omega \rightarrow \mathbb{R}^{n}, R:[0, \infty) \times \Omega \times\left(0, \varepsilon_{0}\right] \rightarrow \mathbb{R}^{n}, \Omega$ an open subset of $\mathbb{R}^{n}, f, g$ and $R$ periodic of period $T$ in the variable $t$,

$$
f^{1}(t, x)=\frac{\partial f}{\partial x} y^{1}(t, x), \quad \text { where } y^{1}(t, x)=\int_{0}^{t} f(s, x) d s .
$$

Of course, $f^{0}, f^{10}$ and $g^{0}$ denote the averaged functions of $f, f^{1}$ and $g$, respectively, defined as in (6). Suppose:

(i) $\partial f / \partial x$ is Lipschitz in $x, g$ and $R$ are Lipschitz in $x$ and all these functions are continuous on their domain of definition;

(ii) $|R(t, x, \varepsilon)|$ is bounded by a constant uniformly in $[0, L / \varepsilon) \times \Omega \times\left(0, \varepsilon_{0}\right]$;

(iii) $T$ is a constant independent of $\varepsilon$; and

(iv) $y(t)$ belongs to $\Omega$ on the interval of time $[0,1 / \varepsilon]$. Then the following statements hold.

(a) In the time scale $1 / \varepsilon$ we have that $x(t)=y(t)+\varepsilon y^{1}(t, y(t))+O\left(\varepsilon^{2}\right)$.

(b) If $f^{0}(y) \equiv 0$ and $p$ is a singular point of averaged system (9) such that

$$
\left.\frac{\partial\left(f^{10}+g^{0}\right)(y)}{\partial y}\right|_{y=p}
$$

is not zero, then there exists a limit cycle $\phi(t, \varepsilon)$ of period $T$ for system (8) which is close to $p$ and such that $\phi(0, \varepsilon) \rightarrow p$ as $\varepsilon \rightarrow 0$.

(c) The stability or instability of the limit cycle $\phi(t, \varepsilon)$ is given by the stability or instability of the singular point $p$ of the averaged system (9). In fact, the singular point $p$ has the stability behavior of the Poincaré map associated to the limit cycle $\phi(t, \varepsilon)$.

\section{Proofs}

In this section we give the proofs of the results presented in Section 1.

Proof of Proposition 1. The characteristic polynomial of the linear part of the Chua system at the origin is

$$
p(\lambda)=-\lambda^{3}+\left(b_{2}-a b\right) \lambda^{2}+\left(b_{2} a b-a b_{1}-1\right) \lambda-a b .
$$

Imposing that $p(\lambda)=-\lambda\left(\lambda^{2}+\omega^{2}\right)$, we obtain $b=b_{2}=0$ and $b_{1}=\left(\omega^{2}-1\right) / a$. So statement (a) follows.

The characteristic polynomial of the linear part of the Chua system at $p_{-}$is given by

$$
p(\lambda)=-\frac{\left(1-b_{2} \lambda+\lambda^{2}\right)\left[2 a_{1} \lambda+a\left(a_{2}^{2}+a_{2} \sqrt{a_{2}^{2}-4 a_{1} b}-4 a_{1} b\right)\right]+2 a_{1} b_{1} a \lambda}{2 a_{1}} .
$$

The proposition follows imposing that $p(\lambda)=-\lambda\left(\lambda^{2}+\omega^{2}\right)$, and that the equilibrium point $p_{-}$exists.

Proof of Theorem 2. If we consider

$$
\left(a, a_{1}, a_{2}, b, b_{1}, b_{2}\right)=\left(\bar{a}_{0}+\varepsilon \alpha_{0}, \bar{a}_{1}+\varepsilon \alpha_{1}, \bar{a}_{2}+\varepsilon \alpha_{2}, \varepsilon \beta_{0}, \frac{\omega^{2}-1}{a}+\varepsilon \beta_{1}, \varepsilon \beta_{2}\right)
$$


with $\varepsilon>0$ a sufficiently small parameter, then the Chua system becomes

$$
\begin{aligned}
& \dot{x}=\left(\bar{a}_{0}+\varepsilon \alpha_{0}\right)\left(\varepsilon \beta_{0} x+z-\left(\bar{a}_{2}+\varepsilon \alpha_{2}\right) x^{2}-\left(\bar{a}_{1}+\varepsilon \alpha_{1}\right) x^{3}\right), \\
& \dot{y}=-z, \\
& \dot{z}=-\left(\varepsilon \beta_{1}+\frac{\omega^{2}-1}{\bar{a}_{0}+\varepsilon \alpha_{0}}\right) x+y+\varepsilon \beta_{2} z .
\end{aligned}
$$

By the rescaling of variables $(x, y, z)=(\varepsilon X, \varepsilon Y, \varepsilon Z)$, system (10) becomes

$$
\begin{aligned}
\dot{X} & =\left(\bar{a}_{0}+\varepsilon \alpha_{0}\right)\left(\varepsilon \beta_{0} X+Z-\varepsilon\left(\bar{a}_{2}+\varepsilon \alpha_{2}\right) X^{2}-\varepsilon^{2}\left(\bar{a}_{1}+\varepsilon \alpha_{1}\right) X^{3}\right), \\
\dot{Y} & =-Z, \\
\dot{Z} & =-\left(\varepsilon \beta_{1}+\frac{\omega^{2}-1}{\bar{a}_{0}+\varepsilon \alpha_{0}}\right) X+Y+\varepsilon \beta_{2} Z .
\end{aligned}
$$

Now we shall write the linear part at the origin of (11) into its real Jordan normal form

$$
\left(\begin{array}{ccc}
0 & -\omega & 0 \\
\omega & 0 & 0 \\
0 & 0 & 0
\end{array}\right),
$$

when $\varepsilon=0$. For doing that we do the linear change of variables $(X, Y, Z) \rightarrow(u, v, w)$ given by

$$
\begin{aligned}
& X=\frac{\bar{a}_{0}(w+\omega v)}{\omega^{2}}, \\
& Y=w-\frac{w}{\omega^{2}}-\frac{v}{\omega}, \\
& Z=u
\end{aligned}
$$

In these new variables, system (11) is written as follows

$$
\begin{aligned}
\dot{u}= & -v \omega+\varepsilon \frac{-\left(\alpha_{0}\left(1-\omega^{2}\right)+\bar{a}_{0}^{2} \beta_{1}\right)(w+\omega v)+\bar{a}_{0} u \beta_{2} \omega^{2}}{\bar{a}_{0} \omega^{2}}-\varepsilon^{2} \frac{\alpha_{0}^{2}\left(\omega^{2}-1\right)(w+\omega v)}{\bar{a}_{0}^{2} \omega^{2}}, \\
\dot{v}= & u \omega-\varepsilon \frac{\left(\omega^{2}-1\right)\left(-u \alpha_{0} \omega^{4}+\bar{a}_{0}^{2}(w+\omega v)\left(\beta_{0} \omega^{2}+\bar{a}_{0} \bar{a}_{2}(w+\omega v)\right)\right)}{\bar{a}_{0} \omega^{5}} \\
& -\varepsilon^{2} \frac{1}{\omega^{7}}\left(\omega^{2}-1\right)(w+\omega v)\left(\alpha_{0} \beta_{0} \omega^{4}+\bar{a}_{0}(w+\omega v)\left(\bar{a}_{2} \alpha_{0} \omega^{2}+\bar{a}_{0} \alpha_{2} \omega^{2}+\bar{a}_{0}^{2} \alpha_{1}(w+\omega v)\right)\right), \\
\dot{w}= & -\varepsilon \frac{-u \alpha_{0} \omega^{4}+\bar{a}_{0}^{2}(w+\omega v)\left(\beta_{0} \omega^{2}+\bar{a}_{0} \bar{a}_{2}(w+\omega v)\right)}{\bar{a}_{0} \omega^{4}} \\
& -\varepsilon^{2} \frac{(w+\omega v)\left(\alpha_{0} \beta_{0} \omega^{4}+\bar{a}_{0}(w+\omega v)\left(\bar{a}_{2} \alpha_{0} \omega^{2}+\bar{a}_{0} \alpha_{2} \omega^{2}\right)\right)}{\omega^{6}} .
\end{aligned}
$$

Writing the differential system (14) in cylindrical coordinates $(r, \theta, w)$ by $u=r \cos \theta, v=r \sin \theta$ and $w=w$ we have

$$
\begin{aligned}
\frac{d r}{d \theta}= & \varepsilon\left(\frac{r \beta_{2} \cos ^{2} \theta}{\omega}-\frac{\bar{a}_{0}\left(\omega^{2}-1\right) \sin \theta(w+r \omega \sin \theta)\left(\bar{a}_{0} \bar{a}_{2} w+\beta_{0} \omega^{2}\right.}{\omega^{6}}\right. \\
& \left.\times \frac{\left.+\bar{a}_{0} \bar{a}_{2} r w \sin \theta\right)}{\omega^{6}}-\frac{\cos \theta\left(w\left(\alpha_{0}+\bar{a}_{0}^{2} \beta_{1}-\alpha_{0} \omega^{2}\right)+r w\left(\bar{a}_{0} \beta_{1}\right.\right.}{\bar{a}_{0} \omega^{3}} \frac{\left.\left.-2 \alpha_{0}\left(\omega^{2}-1\right)\right) \sin \theta\right)}{\bar{a}_{0} \omega^{3}}\right)+O\left(\varepsilon^{2}\right), \\
\frac{d w}{d \theta}= & \varepsilon \frac{r \alpha_{0} \omega^{4} \cos \theta-\bar{a}_{0}^{2}(w+r \omega \sin \theta)\left(\bar{a}_{0} \bar{a}_{2} w+\beta_{0} \omega^{2}+\bar{a}_{0} \bar{a}_{2} r \omega \sin \theta\right)}{\bar{a}_{0} \omega^{5}}+O\left(\varepsilon^{2}\right) .
\end{aligned}
$$

Now we apply the first order averaging theory as described in Theorem 4 of Section 2. In order to do this, we note that (15) satisfies all the assumptions of Theorem 4, where we identify $t=\theta, T=2 \pi, x=(r, w)^{T}$, 
$F(\theta, r, w)=\left(F_{1}(\theta, r, w), F_{2}(\theta, r, w)\right)$ and $f(r, w)=\left(f_{1}(r, w), f_{2}(r, w)\right)$. In short system (15) is the normal form of system (1) in order to apply the averaging theory.

By calculating $f_{1}$ and $f_{2}$, we get

$$
\begin{aligned}
f_{1}(r, w) & =\frac{1}{2 \pi} \int_{0}^{2 \pi} F_{1}(\theta, r, w) d \theta \\
& =\frac{r\left(\beta_{2} \omega^{4}-2 \bar{a}_{0}^{2} \bar{a}_{2} w\left(\omega^{2}-1\right)-\bar{a}_{0} \beta_{0} \omega^{2}\left(\omega^{2}-1\right)\right)}{2 \omega^{5}} \\
f_{2}(r, w) & =\frac{1}{2 \pi} \int_{0}^{2 \pi} F_{2}(\theta, r, w) d \theta \\
& =-\frac{\bar{a}_{0}\left(2 w \beta_{0} \omega^{2}+\bar{a}_{0} \bar{a}_{2}\left(2 w^{2}+r^{2} \omega^{2}\right)\right)}{2 \omega^{5}} .
\end{aligned}
$$

There is only one solution $\left(r^{*}, w^{*}\right)$ for $f_{1}(r, w)=f_{2}(r, w)=0$ satisfying $r^{*}>0$ and this solution is

$$
\begin{aligned}
r^{*} & =\sqrt{\frac{\Gamma}{2 \bar{a}_{0}^{4} \bar{a}_{2}^{2}\left(\omega^{2}-1\right)^{2}},} \\
w^{*} & =\frac{\bar{a}_{0} \beta_{0} \omega^{2}\left(1-\omega^{2}\right)+\beta_{2} \omega^{4}}{2 \bar{a}_{0}^{2} \bar{a}_{2}\left(\omega^{2}-1\right)},
\end{aligned}
$$

since $\bar{a}_{0} \bar{a}_{2} \neq 0,|\omega| \neq 1$ and $\Gamma>0$. We recall that $\Gamma$ is defined in the statement of Theorem 2 .

We note that the Jacobian $(7)$ at $\left(r^{*}, w^{*}\right)$ takes the value

$$
\frac{\beta_{2}^{2} \omega^{4}-\bar{a}_{0}^{2} \beta_{0}^{2}\left(\omega^{2}-1\right)^{2}}{2 \omega^{6}\left(\omega^{2}-1\right)}
$$

and the eigenvalues of the Jacobian matrix

$$
\left.\frac{\partial\left(f_{1}, f_{2}\right)}{\partial(r, w)}\right|_{(r, w)=\left(r^{*}, w^{*}\right)}=\left(\begin{array}{cc}
0 & -\frac{1}{\sqrt{2} \omega^{5}} \sqrt{\bar{a}_{0}\left(\omega^{2}-1\right) \Gamma} \\
-\frac{1}{\omega^{3}} \sqrt{\frac{\bar{a}_{0} \Gamma}{2\left(\omega^{2}-1\right)^{3}}} & \frac{\beta_{2}}{\omega\left(1-\omega^{2}\right)}
\end{array}\right)
$$

are the ones given in $(2)$.

In short, from Theorem 4 we conclude the proof once we show that periodic solutions corresponding to $\left(r^{*}, w^{*}\right)$ provide a periodic solution bifurcating from the origin of coordinates of the differential system (10) when $\varepsilon=0$. Theorem 4 guarantees for $\varepsilon>0$ sufficiently small the existence of a periodic solution corresponding to the point $\left(r^{*}, w^{*}\right)$ of the form $(r(\theta, \varepsilon), w(\theta, \varepsilon))$ such that $(r(0, \varepsilon), w(0, \varepsilon)) \rightarrow\left(r^{*}, w^{*}\right)$ when $\varepsilon \rightarrow 0$. So system (14) has a periodic solution

$$
(u(\theta, \varepsilon)=r(\theta, \varepsilon) \cos \theta, v(\theta, \varepsilon)=r(\theta, \varepsilon) \sin \theta, w(\theta, \varepsilon))
$$

for $\varepsilon>0$ sufficiently small. Consequently, from relation (16) through the linear change of variables (13) system (11) has a periodic solution $(X(\theta), Y(\theta), Z(\theta))$. Finally, for $\varepsilon>0$ sufficiently small system (10) has a periodic solution $(x(\theta), y(\theta), z(\theta))=(\varepsilon X(\theta), \varepsilon Y(\theta), \varepsilon Z(\theta))$ which tends to the origin of coordinates when $\varepsilon \rightarrow 0$. Thus, it is a periodic solution starting at the zero-Hopf equilibrium point located at the origin of coordinates when $\varepsilon=0$. This completes the proof of theorem.

Since the proof of Theorem 3 is very similar to that of Theorem 2, we will omit some steps in order to avoid some long expressions. 
Proof of Theorem 3. Suppose that we have the conditions given in (3) on the parameters of Chua system (1). Then, by a translation of the equilibrium point $p_{-}$at the origin of coordinates, and a rescaling of variables given by $(x, y, z)=(\varepsilon X, \varepsilon Y, \varepsilon Z)$ the Chua system becomes

$$
\begin{aligned}
& \dot{X}=A_{1} X+\left(\bar{a}_{0}+\alpha_{0} \varepsilon+\varepsilon^{2} \xi\right) Z+A_{2} X^{2}+A_{3} X^{3}, \\
& \dot{Y}=-Z, \\
& \dot{Z}=A_{4} X+Y+\varepsilon^{2} \zeta_{2} Z,
\end{aligned}
$$

where

$$
\begin{aligned}
& A_{1}=\varepsilon^{2}\left(1 / 2 \bar{a}_{1}^{2}\right)\left(\bar{a}_{0}\right)\left(-4 \bar{a}_{1}^{2} \zeta_{0}-\alpha_{1} \alpha_{2} \varepsilon \sqrt{-\bar{a}_{1} \zeta_{0}}+2 \bar{a}_{1} \sqrt{-\bar{a}_{1} \zeta_{0}}\left(\alpha_{2}+\varepsilon \xi_{2}\right)+2 \bar{a}_{1} \alpha_{0} \varepsilon\left(-2 \bar{a}_{1} \zeta_{0}+\alpha_{2} \sqrt{-\bar{a}_{1} \zeta_{0}}\right)\right), \\
& A_{2}=\varepsilon^{2}\left(1 / 2 \bar{a}_{1}\right)\left(\bar{a}_{0}\left(3 \alpha_{1} \varepsilon \sqrt{-\bar{a}_{1} \zeta_{0}}+\bar{a}_{1}\left(\alpha_{2}+6 \sqrt{-\bar{a}_{1} \zeta_{0}}+\varepsilon \xi_{2}\right)\right)+\bar{a}_{1} \alpha_{0} \varepsilon\left(\alpha_{2}+6 \sqrt{-\bar{a}_{1} \zeta_{0}}\right)\right), \\
& A_{3}=\varepsilon^{2}\left(\bar{a}_{1} \alpha_{0} \varepsilon+\bar{a}_{0}\left(\bar{a}_{1}+\alpha_{1} \varepsilon\right)\right), \\
& A_{4}=\left(\omega^{2}-1\right)\left[\bar{a}_{0}^{3}-\alpha_{0}^{3} \varepsilon^{3}-\bar{a}_{0}^{2} \varepsilon\left(\alpha_{0}+\varepsilon \xi_{0}+\bar{a}_{0} \alpha_{0} \varepsilon^{2}\left(\alpha_{0}+2 \varepsilon \xi_{0}\right)\right)\right]+\bar{a}_{0}^{4} \varepsilon\left(\beta_{1}+\varepsilon \zeta_{1}\right) .
\end{aligned}
$$

The linear part of (17) at $p_{-}$in the real Jordan normal form when $\varepsilon=0$ is given by (12), and doing also the linear change of variables $(X, Y, Z) \rightarrow(u, v, w)$ given by (13) we write the linear part of system (17) in its real Jordan normal form when $\varepsilon=0$, we obtain the system

$$
\begin{aligned}
\dot{u} & =-\omega v+\varepsilon\left(B_{1} v+B_{2} w\right)+\varepsilon^{2} \zeta_{2} u, \\
\dot{v} & =\omega u+\frac{\varepsilon \alpha_{0}\left(\omega^{2}-1\right) u}{\bar{a}_{0} \omega}+\varepsilon \frac{\omega^{2}-1}{\bar{a}_{0} \omega} B_{3}, \\
\dot{w} & =\frac{\varepsilon \alpha_{0}}{\bar{a}_{0}} u+\frac{\varepsilon^{2}}{\bar{a}_{0}} B_{3},
\end{aligned}
$$

where

$$
\begin{aligned}
& B_{1}=-\frac{\bar{a}_{0}^{3}\left(\beta_{1}+\zeta_{1}\right)-\bar{a}_{0}\left(\alpha_{0}+\varepsilon \xi_{0}\right)\left(\omega^{2}-1\right)-\varepsilon \alpha_{0}^{2}\left(\omega^{2}-1\right)}{\bar{a}_{0}^{2} \omega}, \\
& B_{2}=-\frac{\bar{a}_{0}^{3}\left(\beta_{1}+\zeta_{1}\right)-\bar{a}_{0}\left(\alpha_{0}+\varepsilon \xi_{0}\right)\left(\omega^{2}-1\right)+\varepsilon \alpha_{2}^{2}\left(\omega^{2}-1\right)}{\bar{a}_{0}^{2} \omega^{2}}, \\
& B_{3}=\xi_{0} u-\frac{\bar{a}_{0}^{2}(w+\omega v)\left(2\left(-2 \bar{a}_{1} \zeta_{0}+\alpha_{2} \sqrt{\bar{a}_{1} \zeta_{0}}\right) \omega^{4}-\bar{a}_{0} \bar{a}_{1}\left(\alpha_{2}\right.\right.}{2 \bar{a}_{1} \omega^{6}} \frac{\left.\left.+6 \omega^{2}(w+\omega v) \sqrt{\bar{a}_{1} \zeta_{0}}\right)+2 \bar{a}_{0}^{2} \bar{a}_{1}^{2}(w+\omega v)^{2}\right)}{2 \bar{a}_{1} \omega^{6}} .
\end{aligned}
$$

If we write system (18) in cylindrical coordinates $(r, \theta, w)$ defined by $u=r \cos \theta, v=r \sin \theta$ and $w=w$, after we take as new independent variable the angle $\theta$, and we apply to the system $d r / d \theta$ and $d w / d \theta$ that we obtain the second order averaging method described in Theorem 5 , we get that the function $f=\left(f_{1}, f_{2}\right)$ is identically zero, and that the function $g=\left(g_{1}, g_{2}\right)$ is

$$
\begin{aligned}
g_{1}(r, w)= & \frac{\pi r}{4 \omega}\left(4 \zeta_{2}+\frac{\bar{a}_{0}\left(\omega^{2}-1\right)\left(4 \bar{a}_{0} \bar{a}_{1} w\left(\alpha_{2}+6 \sqrt{-\bar{a}_{1} \zeta_{0}}\right) \omega^{2}\right.}{\bar{a}_{1} \omega^{6}}\right. \\
& \left.+\frac{\left.4\left(2 \bar{a}_{1} \zeta_{0}-\alpha_{2} \sqrt{-\bar{a}_{1} \zeta_{0}}\right) \omega^{4}-3 \bar{a}_{0}^{2} \bar{a}_{1}^{2}\left(4 w^{2}+3 r^{2} \omega^{2}\right)\right)}{\bar{a}_{1} \omega^{6}}\right) \\
g_{2}(r, w)= & \frac{\bar{a}_{0} \pi}{2 \bar{a}_{1} \omega^{7}}\left(4 w\left(2 \bar{a}_{1} \zeta_{0}-\alpha_{2} \sqrt{-\bar{a}_{1} \zeta_{0}}\right) \omega^{4}-2 \bar{a}_{0}^{2} \bar{a}_{1}^{2} w\left(2 w^{2}+3 r^{2} \omega^{2}\right)\right. \\
& \left.+\bar{a}_{0} \bar{a}_{1}\left(\alpha_{2}+6 \sqrt{-\bar{a}_{1} \zeta_{0}}\right) \omega^{2}\left(2 w^{2}+r^{2} \omega^{2}\right)\right) .
\end{aligned}
$$

In order to find solutions $\left(r^{*}, w^{*}\right)$ of $g=0$ we compute a Gröbner basis $\left\{b_{k}(r, w), k=1, \ldots, 20\right\}$ in the variables $r$ and $w$ for the set of polynomials $\left\{\bar{g}_{1}(r, w), \bar{g}_{2}(r, w)\right\}$ where $\bar{g}_{1}=4\left(\bar{a}_{1} \omega^{7} / \pi r\right) g_{1}$ and $\bar{g}_{2}=\left(2 \bar{a}_{1} \omega^{7} / \bar{a}_{0} \pi\right) g_{2}$ and then we will look for roots of $b_{1}$ and $b_{2}$. It is a known fact that the solutions 
of a Gröbner basis of $\left\{\bar{g}_{1}(r, w), \bar{g}_{2}(r, w)\right\}$ are the solutions of $\bar{g}_{1}=0$ and $\bar{g}_{2}=0$, consequently solutions of $g_{1}=0$ and $g_{2}=0$ as well. For more information about Gröbner basis see [33,34].

The Gröbner basis for the polynomials $\left\{\bar{g}_{1}(r, w), \bar{g}_{2}(r, w)\right\}$ in the variables $r$ and $w$ is formed by twenty polynomials. We only use two polynomials of this basis, namely,

$$
\begin{aligned}
G_{1}(r, w)= & 30\left(\omega^{2}-1\right) \bar{a}_{0}^{4} \bar{a}_{1}^{3} w^{3}-15\left(\omega^{2}-1\right) \omega^{2} \bar{a}_{1}^{2} \bar{a}_{0}^{3}\left(\alpha_{2}+6 \sqrt{-\bar{a}_{1} \zeta_{0}}\right) w^{2} \\
& +2 \bar{a}_{0} \bar{a}_{1} \omega^{4}\left(-6 \bar{a}_{1} \zeta_{2} \omega^{2}+\bar{a}_{0}\left(\alpha_{2}^{2}-42 \bar{a}_{1} \zeta_{0}\right.\right. \\
& \left.\left.+15 \alpha_{2} \sqrt{-\bar{a}_{1} \zeta_{0}}\left(\omega^{2}-1\right)\right)\right) w+2 \omega^{6}\left(\bar{a}_{1}\left(\alpha_{2}+6 \sqrt{-\bar{a}_{1} \zeta_{0}} \zeta_{2} \omega^{2}\right)\right. \\
& \left.+\bar{a}_{0}\left(8 \bar{a}_{1} \alpha_{2} \zeta_{0}-\alpha_{2}^{2} \sqrt{-\bar{a}_{1} \zeta_{0}}-12\left(-\bar{a}_{1} \zeta_{0}\right) \sqrt{-\bar{a}_{1} \zeta_{0}}\left(\omega^{2}-1\right)\right)\right)
\end{aligned}
$$

and

$$
\begin{aligned}
G_{2}(r, w)= & \bar{a}_{0} \bar{a}_{1} \omega^{2}\left(6 \bar{a}_{0} \bar{a}_{1} w-\alpha_{2} \omega^{2}-6 \omega^{2} \sqrt{-\bar{a}_{1} \zeta_{0}}\right) r^{2}+2 w\left(2 \bar{a}_{0}^{2} \bar{a}_{1}^{2} w^{2}\right. \\
& \left.-\bar{a}_{0} \bar{a}_{1} w\left(\alpha_{2}+6 \sqrt{-\bar{a}_{1} \zeta_{0}}\right) \omega^{2}+2\left(-2 \bar{a}_{1} \zeta_{0}+\alpha_{2} \sqrt{-\bar{a}_{1} \zeta_{0}}\right) \omega^{4}\right) .
\end{aligned}
$$

Since $G_{1}(r, w)=G_{1}(w)$ is a polynomial of degree 3 in the variable $w$, it is clear that we can have at most three real solutions for $w$ depending on the parameters of the zero-Hopf family. Replacing these three values of $w$ in the second polynomial $G_{2}$ we have six solutions for $r$ of the form $\pm r_{i}^{*}$ for $i=1,2,3$, because $G_{2}(r, w)$ is of the form $P_{1}(w) r^{2}+P_{2}(w)$. However, since $r$ must be positive, we have at most three good solutions for $G_{1}=0$ and $G_{2}=0$. Consequently, we have at most three good solutions for $g=\left(g_{1}, g_{2}\right)=0$ and then, by Theorem 5 and using the same arguments that in the proof of Theorem 2 when we go back through the changes of coordinates, we can have at most three limit cycles bifurcating from the equilibrium point $p_{-}$.

Moreover, if we consider $\alpha_{2}=-6 \sqrt{-\bar{a}_{1} \zeta_{0}}$, then the relations $\left(g_{1}(r, w), g_{2}(r, w)\right)=(0,0)$ provide three solutions given by

$$
\left(r^{*}, w_{ \pm}^{*}\right)=\left(\frac{2 \omega}{\sqrt{15}} \sqrt{\frac{8 \bar{a}_{0} \zeta_{0}\left(1-\omega^{2}\right)-\zeta_{2}}{\bar{a}_{0}^{3} \bar{a}_{1}\left(\omega^{2}-1\right)}}, \pm \frac{\omega^{2}}{\bar{a}_{0} \sqrt{\bar{a}_{1}}} \sqrt{-\frac{4 \bar{a}_{0} \zeta_{0}\left(1-\omega^{2}\right)+2 \zeta_{2} \omega^{2}}{5 \bar{a}_{0}\left(\omega^{2}-1\right)}}\right)
$$

and

$$
\left(r^{0}, w^{0}\right)=\left(\frac{2 \omega}{\sqrt{3}} \sqrt{\frac{4 \bar{a}_{0} \zeta_{0}\left(1-\omega^{2}\right)+\zeta_{2}}{\bar{a}_{0}^{3} \bar{a}_{1}\left(\omega^{2}-1\right)}}, 0\right)
$$

as long as the expressions in the square roots are positives. This shows that three limit cycles can bifurcate simultaneous from the equilibrium $p_{-}$. In a similar way we can produce examples with one, or two limit cycles bifurcating from $p_{-}$. This completes the proof of the theorem.

\section{Acknowledgments}

We thank Pedro T. Cardin and Tiago de Carvalho for their comments which helped us to improve the presentation of this paper.

The first author is supported by the FAPESP-BRAZIL grants 2010/18015-6, 2012/05635-1, and 2013/25828-1. The second author is partially supported by MINECO/FEDER grants MTM2008-03437 and MTM2013-40998-P, AGAUR Grant No. 2014SGR568, ICREA Academia, the grants FP7-PEOPLE2012-IRSES 318999 and 316338, FEDER-UNAB-10-4E-378, and a CAPES grant 88881. 030454/2013-01 do Programa CSF-PVE. 


\section{References}

[1] L.O. Chua, M. Komuro, T. Matsumoto, The double scroll family, IEEE Trans. Circuits Syst. 33 (1986) $1072-1097$.

[2] E.S. Kuetche Mbe, H.B. Fotsin, J. Kengne, P. Woafo, Parameters estimation based adaptive generalized projective synchronization (GPS) of chaotic Chua's circuit with application to chaos communication by parametric modulation, Chaos Solitons Fractals 61 (2014) 27-37.

[3] R. Kiliç, Experimental study on impulsive synchronization between two modified Chua's circuits, Nonlinear Anal. RWA 7 (2006) 1298-1303.

[4] K.W. Lee, S.N. Singh, Robust control of chaos in Chua's circuit based on internal model principle, Chaos Solitons Fractals 31 (2007) 1095-1107.

[5] R. Riaza, Dynamical properties of electrical circuits with fully nonlinear memristors, Nonlinear Anal. RWA 12 (2011) 3674-3686.

[6] J.J. Yan, J.S. Lin, T.L. Liao, Synchronization of a modified Chua's circuit system via adaptive sliding mode control, Chaos Solitons Fractals 36 (2008) 45-52.

[7] J. Llibre, C. Valls, Analytic integrability of a Chua system, J. Math. Phys. 49 (10) (2008) 102701. 9 pp..

[8] B. Rossetto, J.M. Ginoux, Differential geometry and mechanics: applications to chaotic dynamical systems, Internat. J. Bifur. Chaos Appl. Sci. Engrg. 16 (4) (2006) 887-910.

[9] M. Messias, Dynamics at infinity of a cubic Chua's system, Internat. J. Bifur. Chaos Appl. Sci. Engrg. 21 (1) (2011) 333-340.

[10] M. Messias, D.C. Braga, L.F. Mello, Degenerate Hopf bifurcations in Chua's system, Internat. J. Bifur. Chaos Appl. Sci. Engrg. 19 (2) (2009) 497-515.

[11] A. Algaba, M. Merino, F. Fernández-Sánchez, A.J. Rodríguez-Luis, Hopf bifurcations and their degeneracies in Chua's equation, Internat. J. Bifur. Chaos Appl. Sci. Engrg. 21 (2011) 2749-2763.

[12] A. Algaba, E. Freire, E. Gamero, Hypernormal form for the Hopf-Zero bifurcation, Internat. J. Bifur. Chaos Appl. Sci. Engrg. 8 (1998) 1857-1887.

[13] F. Dumortier, S. Ibáñez, H. Kokubu, C. Simó, About the unfolding of a Hopf-Zero singularity, Discrete Contin. Dyn. Syst. 33 (2013) 4435-4471.

[14] J. Guckenheimer, P. Holmes, Nonlinear Oscillations, Dynamical Systems, and Bifurcations of Vector Fields. Revised and Corrected Reprint of the 1983 Original, in: Applied Mathematical Sciences, vol. 42, Springer-Verlag, New York, 1990.

[15] B. Krauskopf, C. Rousseau, Codimension-three unfolings of reflectionally symmetric planar vector fields, Nonlinearity 10 (1997) 1115-1150.

[16] J.L. Moiola, L.C. Chua, Hopf bifurcations and degeneracies in Chua's circuit-a persective from a frequency domain approach, Internat. J. Bifur. Chaos Appl. Sci. Engrg. 9 (1999) 295-303.

[17] J. Guckenheimer, On a Codimension Two Bifurcation, in: Lectures Notes in Math., vol. 898, 1980, pp. 99-142.

[18] M. Han, Existence of periodic orbits and invariant tori in codimension two bifurcation of three dimensional systems, J. Sys. Sci. Math. Sci. 18 (1998) 403-409.

[19] Y.A. Kuznetsov, Elements of Applied Bifurcation Theory, third ed., Spring-Verlag, 2004.

[20] J. Scheurle, J. Marsden, Bifurcation to quasi-periodic tori in the interaction of steady state and Hopf bifurcations, SIAM J. Math. Anal. 15 (1984) 1055-1074.

[21] I. Baldomá, T.M. Seara, Brakdown of heteroclinic orbits for some analytic unfoldings of the Zero-Hopf singularity, J. Nonlinear Sci. 16 (2006) 543-582.

[22] I. Baldomá, T.M. Seara, The inner equation for generic analytic unfoldings of the Zero-Hopf singularity, Discrete Contin. Dyn. Syst. Ser. B 10 (2008) 323-347.

[23] H.W. Broer, G. Vegter, Subordinate Silnikov bifurcations near some singularities of vector fields having low codimension, Ergodic Theory Dynam. Systems 4 (1984) 509-525.

[24] A.R. Champneys, V. Kirk, The entwined wiggling of homoclinic curves emerging from saddle-node/Hopf instabilities, Physica D 195 (2004) 77-105.

[25] J.L. Lagrange, Mécanique Analytique (2 vols.), edition Albert Blanchard, Paris, 1788.

[26] P.S. Laplace, Oeuvres de Laplace, Gauthier-Villars, Paris, 1878.

[27] E.V. Appleton, B. van der Pol, On a type of oscillation-hysteresis in a simple triode generator, Lond. Edinburgh Dublin Philos. Mag. J. Sci. Ser. 6 (43) (1922) 177-193.

[28] P. Fatou, Sur le mouvement d'un système soumis à des forces à courte période, Bull. Soc. Math. France 56 (1928) $98-139$.

[29] N.N. Bogoliubov, N. Krylov, The application of methods of nonlinear mechanics in the theory of stationary oscillations, Publ. 8 of Ukrainian Acad. Sic. Kiev, 1934.

[30] N.N. Bogoliubov, On some statistical methods in mathematical physics, Izv. Akad. Nauk Ukraini. SSSR, Kiev (1945).

[31] J.A. Sanders, F. Verhulst, Averaging Methods in Nonlinear Dynamical Systems, in: Appl. Math. Sci., vol. 59, Springer, 1985.

[32] A. Buică, J. Llibre, Averaging methods for finding periodic orbits via Brouwer degree, Bull. Sci. Math. 128 (2004) 7-22.

[33] W.W. Adams, P. Loustaunau, An Introduction to Gröbner Bases, in: Graduate Studies in Mathematics, vol. 3, American Mathematical Society, 1994.

[34] H. Li, Gröbner Bases in Ring Theory, World Scientific Publishing, 2011. 